Théologiques

Théologiques

\title{
Le silence dans la peinture contemporaine
}

\section{François-Marc Gagnon}

Volume 7, numéro 2, automne 1999

\section{Silence !}

URI : https://id.erudit.org/iderudit/005006ar

DOI : https://doi.org/10.7202/005006ar

Aller au sommaire du numéro

\section{Éditeur(s)}

Faculté de théologie de l'Université de Montréal

\section{ISSN}

1188-7109 (imprimé)

1492-1413 (numérique)

Découvrir la revue

\section{Citer cet article}

Gagnon, F.-M. (1999). Le silence dans la peinture contemporaine. Théologiques, 7(2), 53-77. https://doi.org/10.7202/005006ar

\section{Résumé de l'article}

Rien n'est plus silencieux qu'un tableau. Une longue évolution a conduit àces silences volontaires. On trace ici ce parcours, depuis l'iconographiereligieuse " parlante » en passant par le travail des surréalistes, l'art abstraitet la peinture contemporaine. De la fenêtre sur le monde au tableau-mur lesartistes ont dû lutter pour que leur art ne serve plus les intérêts des puissants,mais qu'il conduise au silence et au questionnement. d'utilisation que vous pouvez consulter en ligne.

https://apropos.erudit.org/fr/usagers/politique-dutilisation/ 


\title{
Le silence dans la peinture contemporaine
}

\author{
François-M arc GAGN ON \\ Professeur d'histoire de l'art \\ Université de M ontréal
}

Au contraire des oeuvres littéraires, les tableaux ne parlent pas. Léonard de Vinci définissait la peinture comme une « poésie muette » et allait jusqu'à recommander à ses élèves qui voulaient représenter l'esprit d'un personnage, de s'attacher à l'observation des mouvements et des gestes des muets, comme si l'on trouvait chez eux moins d'artifice, une expression à l'état pur des états mentaux ${ }^{1}$. Le silence est donc le propre des tableaux et si l'on peut les faire parler comme on dit, en les commentant, ce ne peut être qu'une façon métaphorique de s'exprimer. Laissés à eux-mêmes, les tableaux ne parlent pas.

Ces affirmations qui semblent aller de soi et relever de la pure évidence sont à vrai dire de récentes conquêtes. $M$ ême Léonard qui faisait l'éloge du mutisme de la peinture n'en pensait pas moins qu'elle était aussi « poésie », et donc une forme de langage. D epuis fort longtemps, en christianisme en particulier, c'est plutôt le pouvoir d'instruction des images qui semblait aller de soi. O n connaît la belle formule de saint J ean D amascène : «A près tout, une image est un aidemémoire. Elle est aux illettrés ce qu'un livre est aux lettrés et ce que la parole est à l'ouïe, l'image l'est à la vue ${ }^{2}{ }^{2}$ O n connaît peut-être moins la manière dont nos prêtres missionnaires des débuts de la colonie ont exprimé la même idée. Pour le père Claude Chauchetière, jésuite qui était peintre et qui parlait des images utilisées par les missionnaires pour la conversion des Indiens du Canada au XVII ${ }^{e}$ siècle, elles étaient - la formule est pres-

1. Voir l'édition d'André CH ASTEL Traité de la peinture de L éonard, Paris, Berger-Levrault, 1987, p. 246.

2. O ratio I (PG 94, col. 1237D). 
que léonardienne - «leurs docteurs muets ». ${ }^{3}$ Pour le père Le J eune, un de ses confrères, « ces sainctes figures sont la moitié de l'instruction que l'on peut donner aux Sauvages ». ${ }^{4}$ II est difficile de marquer plus fortement la confiance que l'on mettait dans les potentialités pédagogiques de l'image. Certes, elle ne peut remplacer purement et simplement la parole du missionnaire, mais « ces images [...] parlent desja d'elles mesmes », disait encore le père Le J eune. ${ }^{5}$ Le P. J ean Pierron, qui pratiqua comme le père Chauchetière la peinture missionnaire, $y$ avait même trouver moyen de s'éduquer lui-même dans les langues indiennes qu'il ne maîtrisait pas encore.

...j'ay cet advantage, qu'ils [les Indiens] se servent de Prédicateurs à eux mesmes, \& que ceux qui ne viendroient pas prier par dévotion, y viennent du moins par curiosité, $\&$ se laissent ainsi insensiblement prendre par cet attrait. Enfin j'ay trouvé moy mesme le secret de m'instruire ; car en les entendant raconter nos $M$ ystères, j'apprends beaucoup de la langue, par le moyen de ces images. ${ }^{6}$

Certes, dans tous ces témoignages, on s'exprime au figuré en parlant du discours des images. Il est arrivé cependant qu'on soit allé beaucoup plus loin et qu'on ait cru littéralement à des images capables de parler! La croyance dans les images parlantes ou simplement mouvantes est en effet bien attestée. Et qui plus est elle a été elle-même sujet de tableaux.

\section{Les images parlantes}

L'iconographie des saints nous en fournit quelques exemples. Ainsi I'une des gravures de la Vie de saint Bernard, publiée à Anvers, 1653 par Jacobus N effs (1610-1660) représente le saint échangeant une salutation avec la statue de la Vierge dans le cloître de son abbaye. Comme dans une bande dessinée, I'un dit Ave $M$ aria et l'autre lui répond: Salve Bernarde (Salut Bernard!). O n nous précise dans le

3. N ous citons les Relations des jésuites dans l'édition classique de R. G. THWAITES, The Jesuits Relations and Allied D ocuments, Cleveland, 18961901 et nous y référons par la suite de la manière habituelle : J es. R el., vol. 62, p. 166.

4. Relation du P. Le Jeune, 1637, Jes. Rel., vol. 11, p. 86.

5. Relation du P. Le Jeune, 1633, Jes. Rel., vol. 6, p. 34.

6. Jes. Rel., vol. 52, p. 118. 
titre de la gravure que cela s'est passé alors que saint Bernard était in affligemio , dans un moment d'abattement, de découragement.

Parmi les événements qui contribuèrent à la conversion de saint François (1181 ou 1226), événements qui en général supposent la présence d'un crucifix, il y en a un qui est particulièrement frappant. II se persuada qu'en une occasion au moins, il vit clairement bouger les lèvres du crucifié, et que celui-ci sembla lui adresser la parole. C'est du moins ce que rapporte Thomas de Celano au chapitre 5 de sa L egenda Antiqua et au chapitre 6 de son Tractatus secundus super Vitam Sancti Francisci de Assisi cum miraculis, qui lui s'intitulait «De ymagine crucifixi, que sibi locuta fuit et honore quem ei impendit $\gg{ }^{7}$ Ce genre d'hallucination visuelle et auditive est d'autant moins difficile à croire que nous avons tous expérimenté le phénomène des tableaux qui nous suivent des yeux. II est vrai que celui de les entendre parler est moins commun! Cet appel à l'expérience d'un chacun ne saurait toutefois expliquer à lui seul l'immense influence du récit de Celano. Des miracles analogues allèrent en se multipliant peu après et le nombre de saints personnages à avoir expérimenté pareille vision alla en augmentant dans les siècles qui suivirent. ${ }^{8}$

Le récit des plus improbables miracles de ce genre se trouve souvent sous la plume des écrivains du $X I V^{e}$ et du $X V^{e}$ siècles, y compris des compilateurs comme Caesarius de H eisterbach (1180 -1240) et G autier deCoinci (1177-1236). O n lit dans le D ialogus miraculorum du premier I'histoire un peu salée d'une nonne qui s'était éprise d'un clerc et qui au lieu d'aller se coucher au dortoir comme elle aurait dû le faire, tenta de sortir de l'église. $M$ ais à chacune de ses tentatives, elle trouva la porte fermée par un crucifix. Prenant conscience de sa faute, elle chercha à se faire pardonner au pied d'une statue de la Vierge. $M$ al lui en prit, car

...la statue la gifla et lui dit : « $O$ ù veux-tu aller, ô femmefolle? Retourne à ton dortoir ». Le coup avait été si violent qu'elle tomba à la renverse

7. Voir l'édition des Vies de saint François par Thomas de C elano de H. G. Rosed ALE, Londres, 1904, p. 13 pour la L egenda Antiqua et p. 11 pour le Tractatus secundus.

8. David Freedberg, The Power of Images. Studies in the $\mathrm{H}$ istory and Theory of Response, Chicago/L ondon, The University of Chicago Press, 1989, p. 305. 
et ne put se relever que le matin suivant. II lui avait fait mal, mais il I'avait délivré de toute tentation. A ux grands maux, les grands remèdes!

DeCoinci de son côté, dans ses $M$ iracles de $N$ ostre $D$ ame raconte un fait plus extraordinaire encore. Il y avait, dit-il, une abbayeà $N$ otreD ame magnifiquement ornée de peintures et de sculptures. Les gens avaient même l'habitude de s'arrêter pour contempler le beau portail sculpté et peint qui donnait sur la route. Un moine de l'ordre s'était donné beaucoup de peine pour le peindre « car il estoit moult expert en entailleure». II avait déjà peint une $M$ adoneà l'Enfant au centre du portail et au dessus avait entrepris de peindre un Jugement dernier. Q uand il se fut agi de peindre le diable en Enfer, il le fit si hideux qu'aucune peinture ni sculpture ne fut jamais aussi laides. Vexé, le diable le poussa et le fit tomber de son échafaudage, mais la $M$ adone, aidée de l'Enfant, étendit le bras et l'arrêta dans sa chute. Plusieurs furent témoins du miracle. Le moine descendit del'échelle et son intervention terminée, la Vierge ramena son bras à sa place dans le tableau. ${ }^{9}$ Certes, il ne s'était pas dit un mot dans ce forcément très court épisode - le temps d'une chute - mais ici, les gestes eurent l'éloquence de la parole. Cequ'il importe de relever dans le présent contexte c'est qu'on attribue le mouvement ou la vie à des images.

On pourra trouver particulièrement extravagants des récits de ce genre. $\mathrm{M}$ ais ils posent bien le problème dela relation entrela manière dont un tableau est peint et son efficacité. Gautier de Coinci, par exemple, se montre aussi attentif à la véracité du récit (mentionnant des témoins, par exemple) que portéà faire des considérations sur la composition, les choix formels ( formes tantôt hideuses tantôt si belles qu'elles arrêtent les passants) et le réalisme ex trême des figures (images mouvantes).

Ces récits se rattachent à une forte tradition que l'on pourrait qualifier à la fois de théologique et d'esthétique. II y a très longtemps qu'on prête la vie et la parole à de simples images sculptées ou peintes. Autant au tout début du christianisme, on trouvechez les premiers apologistes de l'Église des condamnations des pratiques païennes qui consistent à prendre de simples idoles pour des êtres vivants, ${ }^{10}$ autant dès l'époque byzan-

9. M iracles de N ostre $D$ ame, éd. de J ean M iélot, 1885 ; citée par David FREeDberG, op. cit., p. 307.

10. Voir par exemple, Lactance, D ivinae institutiones, I.22.13 dans E. H. WARM IN GTON, Vetera L atina, London, Loeb Classical Library, 1935, vol. 3 , p. 166 , nos 524-529. 
tine, on accepta plus volontiers les récits de miracles impliquant des images parlantes ou au moins douées de mouvement. Ainsi dans plusieurs psautiers byzantins dits « marginaux » on peut voir une image du Christ étendant la main hors du cadre du tableau où il est peint, pointant vers les croyants, ou, le plus souvent, vers D avid lui-même. II semblealors être en conversation avec eux ou il les bénit « pour vrai ».

De même que Dieu s'était incarné dans la personne de son Fils et que I'on était autorisé de représenter la divinité sous des traits humains, de même il allait de soi que certaines images saintes fussent dotées de traits humains, comme du mouvement et de la parole. En réalité, ces images parlantes poussaient la logique del'incarnation jusque dans le domaine iconographique et servaient en dernière analyse la doctrine elle-même. Ce n'est pas le lieu ici de reprendre le débat sur les images auquel mettra fin temporairement le deuxième Concile de $N$ icée, 787 et qui sera repris au concile de Trente, 1545-1563 à cause des critiques de la R éforme contre le culte catholique des images. Chaque fois on y réaffirma la distinction entre le culte de dulie adresséaux créatures représentées et le culte de latrie, adressé à Dieu seul. Chaque fois on y proclama, contre les tentations iconoclastes, l'attachement de l'Église de Rome au culte des images, miraculeuses ou pas.

II nefaudrait pas penser que la croyance aux images vivantes appartienne seulement au passé. David Freedberg cite le cas d'une icône que 5,000 personnes par jour venaient voir en 1986 dans l'église albanaise de C hicago parce qu'elle émettait de ses yeux et de ses mains une substance huileuse. ${ }^{11} \mathrm{~N}$ 'est-ce pas à peu près dans le même temps, qu'on a vu ici au Q uébec, des foules attirées par une image de la Vierge des yeux de laquelle s'échappait une substance graisseuse qui faisait penser à des larmes? O n eut tôt fait de crier à l'imposture, au mercantilisme, à la simonie. O n ne devrait pas prendre à la légère le fait que des milliers de gens étaient prêts à croire ce qu'on leur disait à ce sujet et après une visite sur les lieux, où parfois ils avaient fait la queue durant des heures

11. II renvoie au New York Times, 22 décembre 1986: «The Greek Orthodox Church has no plans to analyse the moisture which Father Koufos described as a very thin oily sw eet substance very similar to the chrism we use to baptize children or unction for the sick. To further analyse it would be almost blasphemy, he said. The Archdiocese thinks they should not subject it to scientific analysis which is not a very religious procedure ». 
tant l'affluence était grande, revenaient chez eux convaincus d'avoir vu la Vierge pleurer. Le mépris des intellectuels pour ce genre de phénomène montre seulement qu'ils sous-estiment le pouvoir des images. Ils lefont d'ailleurs hypocritement, car ces mêmes intellectuels cèdent aisément au charme des images dans d'autres contextes.

\section{Les automates}

Q ue dire en effet de l'intérêt des surréalistes pour les mannequins, les poupées et surtout les automates, sinon qu'il s'agit d'une forme pour ainsi dire sécularisée de fascination pour les images mouvantes et parlantes? O n sait qu'ils ont été spécialement intrigués par le J eune écrivain, fameux automate construit par Pierre J acquet-D roz en 1774, représentant un enfant aux cheveux bouclés, vêtu à l'ancienne, avec jabot et manchettes de dentelles, assis sur un tabouret, absorbé dans son travail d'écriture, la plume d'oie à la main. Aux dires de Benjamin Péret, cette poupée mécanique écrivait toujours le mot " merveilleux ${ }^{12}$ suggérant que pour les surréalistes, le merveilleux se produit non pas lorsque la cause rationnelle d'un événement nous échappe mais simplement lorsqu'elle nous est cachée. Aussi, quand on reproduisit cet automate dans la revue $M$ inotaure en 1933, on illustra le « mécanisme des yeux », en montrant ouvert son crâne de métal, et le « mécanisme de L'Écrivain », en le figurant de dos, de manière à faire voir les manettes et les roues à engrenages responsables de son fonctionnement. Le « merveilleux » ne coïncide pas purement et simplement avec le miraculeux des anciennes images. Le Jeune écrivain est aussi une sorte d'H omme machine, pour reprendre le titre du livre que de $L$ a $M$ ettrie avait publié en 1748 . L e J eune écrivain est une merveille, mais c'est aussi une mécanique!

$\mathrm{N}$ otre propre fascination pour les robots de la science fiction est un direct héritage de l'intérêt des surréal istes pour les automates. Tous les enthousiastes de Star Trek connaissent Data, l'androïde dépourvu d'émotions, mais plein de curiosité et de désenchantement pour la condition humaine.

12. Benjamin PÉRET, «Au paradis des fantômes», Minotaure 3-4, 14 décembre 1933, p. 35. 
Freud a tenté d'aller aux racines inconscientes de cette fascination pour ce genre d'images dans son fameux essais de 1919, D as U nheimliche, L'inquiétante étrangeté. On éprouverait ce sentiment $d^{\prime}$ « inquiétante étrangeté » à la vue de « personnages de cire, de poupées artificielles et d'automates ». Freud citait en exemple L'H omme au sable, un conte de E. T. A. H offmann. O $n$ y racontait que l'étudiant $\mathrm{N}$ athanaël acheta à l'opticien ambulant Coppola une longue-vue de poche qui lui permit d'épier l'appartement du professeur Spalanzani, situé de l'autre côté de la rue. $\mathrm{M}$ ettant I'oeil à l'objectif, $\mathrm{N}$ athanaël aperçut la fille de ce dernier, O lympia, belle comme le jour, bien que mystérieusement laconique et immobile. II en tomba aussitôt amoureux, en oubliant sa propre fiancée, Clara. M ais Olympia n'était qu'un automate fabriqué par Spalanzani et dans lequel Coppola avait inséré des yeux vivants.

La scène d'horreur sur le thème des yeux arrachés ou fixés tout sanglants dans le corps de l'automate, est assez explicite pour que Freud évoque à son propos sans crainte d'être contredit le complexe de castration, les yeux tenant la place des organes génitaux. $M$ ais il dit aussi que ce sentiment d'unheimliche s'enracine en réalité dans le narcissisme primaire, par le biais de l'idée du double.

...le double était à l'origine une assurance contre la disparition du moi, un « démenti énergique de la puissance de la mort » ( 0 . Rank), et il est probable que l'âme « immortelle » a été le premier double du corps. La création d'un tel dédoublement pour se garder de l'anéantissement a son pendant dans une mise en scène de la langue du rêve qui aime exprimer la castration par redoublement ou multiplication du symbole génital ; dans la culture des anciens Égyptiens, elle motive l'art de modeler l'image du défunt dans une matière durable. $M$ ais ces représentations ont poussé sur le terrain de l'amour illimité de soi, celui du narcissisme primaire, lequel domine la vie psychique de l'enfant comme du primitif. Avec le dépassement de cette phase, le signe dont est affecté le double se modifie; d'assurance de survie qu'il était, il devient l'inquiétant [unheimlich] avant-coureur de la mort. ${ }^{13}$

Entendu de cette façon, le concept du double relève du déni inconscient de la mort. O n sait que, toujours d'après Freud,

13. Sigmund FREUD, L'inquiétante étrangeté et autres essais, trad. de I'allemand par Bertrand Féron, Paris, Gallimard, 1985, p. 236-7. 
« personne, au fond, ne croit à sa propre mort ou, ce qui revient au même: dans l'inconscient, chacun de nous est persuadé de son immortalité $\gg .{ }^{14}$ Cette persuasion est aussi celle de la foi et on peut se demander si la croyance dans l'au-delà n'a pas contribué à atténuer le sentiment d'étrangeté que n'aurait pas manqué de produire les images vivantes dont nous parlions au début, si elles avaient été perçues en dehors de son rayonnement.

Les surréalistes n'avaient cure des images parlantes chrétiennes, mais ils cherchaient dans l'image du double, spécialement du mannequin et de l'automate, la résolution d'une des grandes contradictions de l'existence, la plus importante à vrai dire, celle de la vie et de la mort.

Tout porte à croire, écrivait Breton dans le second M anifeste du surréalisme, qu'il existe un certain point del'esprit d'où la vie et la mort, le réel et l'imaginaire, le passé et le futur, le communicable et l'incommunicable, le haut et le bas cessent d'être perçus contradictoirement. Or, c'est en vain qu'on chercherait à l'activité surréal iste un autre mobile quel'espoir de détermination de ce point. ${ }^{15}$

Je ne connais pas d'illustration plus vive et plus efficace de ce que Breton avançait ici que le texte hallucinant de précisions et de délire de Raymond R oussel, auteur admiré des surréal istes, intitulé L ocus Solus, qui décrit une promenade dans le parc entourant la villa du professeur $M$ artial Canterel. Ce dernier, inventeur du «vitalium » et de la « résurrectine » avait su redonner vie pour un moment à une série de huit personnages plus ou moins historiques que les promeneurs pouvaient contempler à loisir derrière une vitre. Ils les voyaient revivre des scènes caractéristiques de leur existence passée et écoutaient les explications de leurs faits et gestes données imperturbablement par $C$ anterel. M ême muets, les cadavres ressuscités par Canterel étaient en réalité des automates parfaits, dont l'animation, comme dans le cas du poète Gérard Lauwerys, arrivait à consoler un court instant sa veuve et son fils, ou à faire revivre une cérémonie aux époux bretons Le $M$ ao, etc. II

14. Sigmund FREUD, «Considérations actuelles sur la guerre et sur la mort (1915) », dans Essais de psychanalyse, Paris, Payot, 1981, p. 26.

15. André BRETON, «Second manifeste du surréalisme, 1930 » dans M anifestes du surréalisme, Paris, J ean-J acques Pauvert éditeur, 1962, p. 154. 
va sans dire que le sentiment d' unheimlichkeit est ici poussé à un paroxysme rarement atteint en littérature contemporaine.

\section{U ne « muraille de peinture »}

Les personnages de R oussel évoluaient dans une sorte de « cage des ressuscités » ou encore dans une « glacière ». On les voyait derrière une vitre et donc comme en autant de vitrines. Les mannequins surréalistes faisaient souvent partie de véritables scènes, comme ceux que l'on pouvait voir à travers les vitres du Taxi pluvieux de Dali lors de l'Exposition Internationale du Surréalisme présentée à la Galerie des beaux-arts, à Paris, en 1938. Breton n'a jamais récusé le grand paradigme de la peinture comme fenêtre. Tout au plus, s'interrogeaitil sur ce qui est donné à voir par cette fenêtre.

...il m'est impossible, écrit-il dans la préface de son grand recueil d'écrits sur l'art qu'est Le Surréalisme et la Peinture, de considérer un tableau autrement que comme une fenêtre dont mon premier souci est de savoir sur quoi elle donne, autrement dit si, d'où je suis, «la vue est belle», et je n'aime rien tant que ce qui s'étend devant moi à perte de vue. ${ }^{16}$

Or ce paradigme remonte au D e Pictura de L eon Battista A lberti, écrit en 1435. Expliquant ce qu'il fait quand il peint, Alberti déclarait en effet : " M on premier acte, quand je veux peindre une superficie, est de tracer un rectangle, de la grandeur qui convient, en guise de fenêtre ouverte où je puisse voir le sujet [ historia] ». ${ }^{17}$ Fidèles à leur manque d'intérêt pour les renouvellements purement formels de la peinture, les surréalistes ne récusèrent pas les vues d'A lberti et continuèrent à concevoir le tableau comme une fenêtre ouverte, il est vrai sur le monde du dedans. $M$ ais tant qu'on s'en tiendra à ce paradigme de la fenêtre, la peinture aura quelque chose à dire : une historia à raconter. Le silence se fera en peinture qu'à une seule condition. II faudra accepter de fermer la fenêtre.

16. André BRETON, Le Surréalisme et la Peinture, Paris, Gallimard, 1965, p. 2-3.

17. Traduction de Philippe HAM OU, La vision perspective (1435-1740), Paris, Payot, 1995, p. 74. Pour une analyse classique du texte d'A lberti, voir E. PAN OSKY, La perspective comme forme symbolique, Paris, M inuit, 1975. 
Curieusement, on trouve cette idée du tableau comme fenêtre mise en question pour la première fois dans la fameuse nouvelle de Balzac, Le Chef-d'oeuvre inconnu, qui comme on sait met en scène le peintre Frenhofer à la poursuite du tableau parfait et qui aboutit, après dix ans de travail acharné, à un tableau incompréhensible. " Je ne vois que des couleurs confusément amassées et contenues par une multitude de lignes bizarres qui forment une muraille de peinture », se serait écrié le grand peintre français, $\mathrm{N}$ icolas Poussin, également mis en scène dans la nouvelle de Balzac, devant le tableau. U ne muraille de peinture! Le mot est lâché. Au paradigme de la fenêtre qui donne sur le réel se substitue celui du mur qui la bouche et ne donne à voir que des couleurs et des lignes. Dans « ce chaos de couleur », un seul élé ment de réal ité aurait été sauvé : « le bout d'un pied nu ». " IIs [Poussin et Pourbus] restèrent pétrifiés d'admiration devant ce fragment échappé à une incroyable, à une lente et progressive destruction ».

En réalité, ce que Frenhofer avait « détruit » dans son tableau, c'était une forme de communication, qui ressemblait au discours. Tant que le spectateur peut nommer ce qu'il voit, comme c'est le cas d'un tableau figuratif, il reste dans l'orbite familière du discours. Confronté à une « muraille de peinture », il perd la sécurité du monde verbal et entre dans le domaine du non verbal, du silence. Certes, dans le tableau de Frenhofer, il restait encore un petit pied capable de dire quelque chose, mais c'était un mot sans phrase, un simple fragment de la femme cachée « là-dessous ». Ce que le vieux peintre imaginaire de Balzac avait « construit » par contre, c'était le premier tableau à ne plus tenir de discours, le premier tableau silencieux.

O n sait que Z ola reprendra ce thème dans L'O euvre, en mettant en scène un personnage qui fait penser à Cézanne. Lui aussi présentera son héros, Claude Lantier, comme un raté, sinon un fou et l'un et l'autre héros finissent de la même façon : par la folie ou le suicide. En réalité cette référenceà Cézanneest tout à fait pertinenteà notre propos. Émile Bernard a raconté que les larmes montaient aux yeux du vieux Cézanne quand on lui parlait de Frenhofer, "quelqu'un par qui il était devancé dans la vie, dont l'âme était prophétique [ et qui] I'avait deviné ». ${ }^{18} \mathrm{En}$ ne recouvrant pas complètement ses toiles de pigments, notait Walter

18. Émile Bern ARD, Souvenirs sur Paul Cézanne, Paris, M ercure deFrance, 1925. 
Benjamin, ${ }^{19}$ en laissant voir ici et là la trame de celle-ci, Cézanne rendait déjà la peinture à son essence de surface et donc de paroi verticale. Cézanne eut recours à plusieurs autres moyens pour indexer la surface du tableau. Le fameux rabattement des tables de ses natures mortes dans le plan du tableau en est un, car il crée un compromis entre les données de la sensation (la tri-dimensionnalité de la table dans l'espace réel) et la réal ité de la toile, pure surface sur laquelle le peintre travaille. Souvent Cézanne s'ingéniait égal ement à réduire les indices de spatialisation d'une manière telle qu'on a de la peine à situer les objets dans l'espace. O ù s'arrête le mur, où commence le sol? Et cette partie de cartes se passe-t-elle à l'extérieur ou à l'intérieur? Enfin, même sa manière de peindre par petites touches obliques, indépendantes du sujet traité, semble renvoyer à la surface picturale comme telle, sans lien avec le monde tridimensionnel qu'il a voulu représenter. C etteindexation dela surface chez C ézanne annoncedonc le passage à un nouveau paradigme del'art, pressenti par Balzac.

Picasso, peut-être à l'initiative de Blaise Cendrars, s'intéressa aussi à la nouvel le de Balzac. C'est en 1931, donc exactement cent ans après sa publication et avant de s'installer lui-même dans la maison où commence le conte, rue des Grands-A ugustins et où il peindra G uernica, que Picasso donna à A mbroise Vollard une série d'oeuvres sur papier inspirées du texte de Balzac. Vollard s'empressa de rééditer le Chefd'oeuvre inconnu orné des 13 eaux-fortes et de 67 bois gravés de Picasso. On pouvait y suivre les hauts et les bas de sa réflexion sur les rapports triangulaires du peintre, du modèle et du tableau, suggérant des substitutions troublantes de l'un à l'autre. En fait, c'est bien plutôt dans l'oeuvre antérieure de Picasso qu'il faut chercher sur le plan formel (et non simplement du contenu comme dans la suite des gravures données à Vollard) une mise en oeuvre de l'intuition de Balzac. Dans les papiers collés, l'intrusion de morceaux empruntés à la réalité (feuille de musique, articles de journaux, aiguille, corde...) ramène la pensée au sentiment d'une surface susceptible de recevoir des objets ${ }^{20}$ et fait passer au second rang le sujet [historia] du tableau.

19. Walter BENJAMIN, «Peinture et graphisme», La part de l'oeil, no 6, 1990.

20. Voir Christine PoGGI, «Frames of Reference: Table and Tableau in Picasso's Collages and Constructions », A rt Journal, vol. 47, no 4 (hiver 1988), p. 311-2. 
Avec Cézanne, Picasso, nous nous éloignons de la tradition surréaliste. A ussi bien, en restant attaché au paradigme de la fenêtre, la peinture surréaliste montrait qu'elle n'avait cure de ce travail formel qui faisait de la peinture contemporaine une aventure unique au pays du silence. II lui fallait des sujets, des spectacles, du « merveilleux ». Une seule exception à ce combat d'arrière-garde : R ené $M$ agritte, qui tout en restant fidèle aux moyens de la peinture la plus platement naturaliste, proposa une critique radicale du paradigme de la fenêtre. $D$ ans $L$ a Clef des champs, 1933, il semble paraphraser de si près le texte d'Alberti qu'il est difficile de penser qu'il ne l'ait pas fait consciemment. Le tableau représente une fenêtre sur la vitre de laquelle un peintre, peignant point par point ce qu'il voyait, aurait suivi à la lettre le conseil d'A lberti. M ais, malheureusement la vitre s'est brisée. Les fragments tombés au sol permettent tout de même de reconnaître en quoi avait consisté l'effort du peintre : reproduire le plus fidèlement possible un paysage insignifiant. L'oeuvre s'intitule, avons-nous dit, La Clef des champs. La peinture nepeut espérer prendre «la clef des champs » qu'à la condition de se libérer du vieux paradigme de la fenêtre, de briser la vitre pour la remplacer par une surface peinte. D'autres tableaux de M agritte, comme L'appel des cimes, 1942 qui laissent voir une immense pan de montagne dont l'un des sommets se change en tête d'aigle suggère qu'il faut substituer une paroi à la fenêtre si I'on veut libérer la peinture de ses anciennes fonctions verbales. Sans ce nécessaire changement, le tableau ne peut prendre son envol comme tableau. $\mathrm{N}$ on seulement on n'arrivera pas à le détacher de la réalité qu'il représente, on sera toujours assujetti à ne traiter que ce qui peut paraître à la fenêtre.

$M$ agritte tient une position paradoxale dans cette réflexion sur la libération de la peinture. II le fait tout en s'en tenant à la figuration la plus directe et par son truchement. M agritte ruine les apparences par les apparences mêmes. Sa stratégie est celle du cheval de Troie. Sans paraître faire la moindre concession à l'abstraction, il discrédite la figuration par la figuration. On sait qu'il ira jusqu'à consacrer un tableau entier à une pipe avec l'inscription : «Ceci n'est pas une pipe», pour bien marquer qu'une image de la réalité n'est pas la réalité. ${ }^{21}$ Alors pourquoi y

21. Du moins c'est l'un des sens de cet intriguant tableau. Voir M ichel FoUCAULT, Ceci n'est pas une pipe, M ontpellier, Fata M organa, 1973 pour une réflexion d'ensemble. 
tenir? Les peintres contemporains qui iront encore plus loin dans le sens pressenti par Balzac et indiqué par Cézanne, Picasso ou M agritte seront non seulement des peintres non figuratifs, mais des peintres ayant adoptés le système de la grille.

\section{La grille}

O n entend par grille tout système de structuration du tableau qui a recours à une forme ou l'autre de carrelage, damier, quadrillé, mise au carreau, etc. II s'agit d'un développement de l'idée de mur, mais en insistant sur la répartition uniforme des éléments sur la surface picturale, à l'absence de points de focalisation et de hiérarchie entre les éléments (all overness), encourageant à lire la surface comme une synecdoque, puisque la partie y vaut pour le tout. II va sans dire que définie ainsi, la grille est une abstraction. Elle a donnée lieu à des présentations et des exploitations les plus variées selon les artistes, depuis les carrelages de M ondrian (Composition dans le damier avec couleurs claires et avec couleurs foncées, 1919) jusqu'aux M icrochromies de Fernand Leduc (Ensemble de Cycles de vie, 1992) en passant par les damiers de Borduas (le grand Symphonie en damier blanc ou Symphonie 2 , 1957, qui appartenait à la collection de R enée Borduas et qui est maintenant au $M$ usée des beaux-arts de $M$ ontréal) ou les quadrillés discrets d'A gnes $M$ artin, une peintre d'origine canadienne ${ }^{22}$ dont on vient de reconnaître l'importance aux États-Unis (L eaf, 1965 de la collection de $M$. et de M me Daniel W. Dietrich II).

Ce n'est pas tout de dire que la grille affirme la surface picturale pour elle-même. Elle ferme la fenêtre de l'art ancien et nous dérobe le spectacle du monde, introduisant un silence non encore expérimenté en peinture. $\mathrm{N}$ ul mieux que Rosalind Krauss a su exprimer cette dimension de l'art d'aujourd'hui.

In the early part of this century there began to appear, first in France and then in R ussia and in $\mathrm{H}$ olland, a structure that has remained emblematic of the modernist ambition within the visual arts ever since. Surfacing in pre-War cubist painting and subsequently becoming ever more stringent and manifest, the grid announces, among other things, modern art's will to silence, its hostility to litterature, to narrative, to discourse. As such, the grid has done its job with striking efficiency. The barrier it has lowe-

22. Elle est née à M aklin en Saskatchewan en 1912. 
red between the arts of vision and those of language has been almost totally successful in walling the visual arts into a realm of exclusive visuality and defending them against the intrusion of speech. The arts, of course, have paid dearly for this success, because the fortress they constructed on the foundation of the grid has increasingly become a ghetto. Fewer and fewer voices from the general critical establishment have been raised in support, appreciation, or analysis of the contemporary plastic arts. ${ }^{23}$

On aurait mal compris le sens de ces dernières remarques de K rauss si on en concluait que son intention était de grossir les rangs de ceux qui reprochent à l'art d'aujourd'hui son silence, et voient dans son hermétisme le signe d'un refus de communiquer avec le public. Cesilence gagné de hautes luttes et parfois au prix d'une atroce solitude est un bien trop précieux à l'artiste moderne pour qu'il accepte d'y renoncer. II y va de sa liberté intérieure. Krauss a noté que les premiers recours à la grille en art moderne pouvaient se réclamer des R omantiques et paradoxalement de leur manière de traiter la fenêtre. Ainsi quand Georg Friedrich Kersting (1785-1847) représente le grand peintre romantique allemand Caspar D avid Friedrich dans son atelier, c. 1811, il le montre tout absorbé dans la contemplation de sa toile, tournant le dos à la fenêtre dont, de toute manière, les volets sont clos à hauteur de la vue. Bien plus l'atelier est complètement vide, les seuls objets accrochés au mur étant deux palettes $\mathrm{d}^{\prime}$ artiste et une règle. Kersting semble avoir voulu illustrer de cette manière certains propos qu'on attribuait au maître. «Q uand un peintre ne peut faire rien d'autre qu'imiter platement la nature, ou plutôt, l'aplatir et la tuer en l'imitant, il ne vaut pas mieux qu'un singe vaniteux ». Ou encore : «La tâche de l'artiste n'est pas seulement de représenter fidèlement l'air, l'eau, les rochers et les arbres, mais d'y refléter son âme et sa sensibilité ». "Toute grande oeuvre d'art est conçue à un moment sanctifié et néà une heure bénie, souvent à l'insu de la perception consciente de l'artiste, sortie du plus profond de son cœur $\gg .{ }^{24} A$ ussi bien la fenêtre qui paraît dans le tableau de Kersting ne donne à voir qu'un coin de ciel couvert de nuages, derrière le cruciforme parfait de ses montants. Cette fenêtre, c'est la grille réduite à sa plus simple expression.

23. « Grids » dans R osalind E. KRAUSS, The $O$ riginality of the Avant-G arde and $O$ ther M odernist M yths, Cambridge/Londres, The M IT Press, 1994, p. 9 24. Cité par Hubert SCHRADE, German Romantic Painting, New York, Harry N. A brams, Inc., 1977, p. 80. 
Il y a à l'origine de l'art moderne et spécialement de l'art abstrait un profond besoin de faire taire le bruit extérieur pour écouter la voie intérieure, «du plus profond de son coeur ». Des Romantiques aux premiers abstraits la filiation sur ce point est parfaite. Q ue l'on pense à Kandinsky publiant D u Spirituel dans I'art en 1912, à M ondrian lisant le théoso phe hollandais M . H . J. Schoenmaekers, auteur de H et $\mathrm{N}$ ieuw e Werel beeld ( $\mathrm{L}$ a N ouvelle I mage du M onde ), publié en 1915, à $M$ alevitch transposant l'anomisme des mystiques russes dans ses prises de positions « suprématistes », toujours le même besoin spirituel se trouve exprimé. Toujours il semble impliquer une mise au second rang du respect des apparences extérieures, sinon leur complète abolition. K andinsky a raconté comment fut déterminante de ce point de vue une expérience qu'il fit dans son atelier de $M$ unich.

... alors que je vivais déjà à $M$ unich, je fus ravi un jour par une vue tout à fait inattendue dans mon atelier. C'était I'heure du jour déclinant. A près avoir travaillé sur une étude, je venais de rentrer chez moi avec ma boîte de peinture...lorsque j'aperçus un tableau d'une indescriptible beauté baigné de couleurs intérieures. J e commençai par me renfrogner, puis me diriger droit vers cette œuvre énigmatique dans laquelle je ne voyais rien d'autre que des formes et des couleurs et dont le sens me restait incompréhensible. Je trouvai instantanément la clé de l'énigme: c'était un de mes tableaux posé de côté contre un mur. Le jour suivant, je voulus reproduire l'impression de la veille à la lumière du jour, mais je n'y parvins qu'à demi ; même de côté, je reconnaissais sans cesse les objets, et il y manquait le subtil glacis du crépuscule. Je savais à présent très exactement que l'objet était nuisible à mes tableaux. ${ }^{25}$

Il s'agissait donc de se libérer del'objet, si l'on voulait atteindre au « spirituel dans l'art ». L'expérience de K andinsky révélait non seulement que l'objet n'était pas nécessaire pour produire l'effet radieux qui l'avait frappé, mais qu'il pouvait même lui nuire. L'artiste ne pouvait espérer entendre sa voix intérieure qu'en abolissant les objets ou, si I'on veut, en réduisant au silence le discours des objets.

Cette conviction persistera bien au-delà de l'époque des pionniers. Comme R obert R osenblum l'a montré, ${ }^{26}$ on en trouve des traces jusque

25. Cité par Ulrike BeCKS-M ALORNY, Wassili Kandinsky 1866-1944. Vers I'abstraction, Taschen, 1994, p. 31-32.

26. Robert ROSENBLUM, Modern Painting and the Northern Romantic Tradition. Friedrich to Rothko, Londres, Thames and H udson, 1974. 
dans l'expressionnisme abstrait américain, dans l'énergie rendue visible par Pollock, dans l'intransigeance de liberté de Clyfford Still, dans la notion de sublime chez Barnett $\mathrm{N}$ ew man, dans l'ouverture totale au tragique chez R othko. Celien avec le spirituel ne sera rompu qu'avec les $M$ inimalistes, quand Donald Judd déclarera son intention de ne faire que des « specific objects », vides de tout signe de sa présence dans leur fabrication. À la limite, le rôle de l'artiste ne consistera plus qu'à concevoir mentalement l'oeuvre qui, elle, sera fabriquée à distance, par un tiers, voire en série. Une oeuvre fabriquée en série se distingue en effet d'une oeuvre originale et ses copies, en ce que l'oeuvre fabriquée en série ne comporte pas d'original. De cette double manière, Judd voudra effacer toute présence physique del'artiste de son oeuvre, présence dont le geste et sa trace avaient toujours paru la condition minimale pour qu'on puisse parler d'oeuvre d'art. M ais cette évacuation de la subjectivité de l'oeuvre d'art, compréhensible après une période qui lui avait peut-êtrefait une trop grande place, ne devait pas durer. Bientôt l'esprit reprenait ses droits, tant il est vrai que le mouvement amorcé par la peinture abstraite au début du siècle est irréversible.

En évacuant de son sein l'anecdote, le mythologique et le religieux, en refusant de servir à des fins d'illustration d'une pensée exprimée en mots, la peinture contemporaine, celle qui a substitué le paradigme du mur à celui de la fenêtreet qui a cherché sa structure dans la grilleplutôt que dans la perspective, a incarné pour un temps l'espoir d'une grande libération de l'esprit humain, comme le disait $M$ al evitch.

Toute la peinture passée et actuelle avant le suprématisme ( sculpture, art verbal, musique) a été asservie par la forme de la nature et attend sa libération pour parler dans sa proprelangue et ne pas dépendre de la raison, du sens, de la logique, de la philosophie, de la psychologie, des différentes lois de la causalité et des changements techniques de la vie. ${ }^{27}$

N ous l'avons vu, Kandinsky parlait aussi de libérer la peinture de l'esclavage de l'objet. M alevitch allait plus loin. Libérée de toute fonction narrative, la peinture devenait un moyen d'expression autonome.

27. J ean-Claude et Valentine M ARCADÉ avec la collaboration de Véronique SCHILTZ, K. S. M alévitch. D e Cézanne au suprématisme. Tous les traités parus de 1915 à 1922, Lausanne, L'Âge d'homme, 1974, p. 37. 
L'art n'a plus à servir ni l'État ni l'Église. L'art netient plus à illustrer I'histoire des moeurs. II ne veut rien avoir avec l'objet et est convaincu qu'il peut exister par lui-même et pour lui-même...sous sa forme pure, le suprématisme ${ }^{28}$.

C'est en effet une des grandes raisons d'être de l'art abstrait de proposer une forme d'art qui ne soit pas récupérable du point de vue idéologique par le pouvoir. Q ue peuvent faire les pouvoirs politiques de tableaux qui ne représentent rien ou dont le symbolisme est tellement universel qu'il s'accommode de toutes les causes et d'aucune en particulier sinon de s'en détourner?

D onc, en prenant ses distances avec la tradition, en se permettant de soumettre ses « évidences » à un nouvel esprit critique, de questionner ses postulats, de refuser de suivre tout programme iconographique imposé, fut-ce par l'Église ou tout autre pouvoir, la peinture apprenait à ne plus prêter sa voix à personne, sinon à se taire.

Peut-être Balzac croyait-il que Frenhofer s'était enfermé dans les murs de sa propre folie et considérait son dernier tableau comme la marque d'un esprit dérangé. II est question du « prétendu tableau », de « couleurs confusément amassées et contenues par une multitude de lignes bizarres », d'une toile où il n'y a « rien ». Pourtant un court dialogue entre Pourbus et Poussin, les deux visiteurs de Frenhofer semble suggérer autre chose.

- Là, reprit Pourbus en touchant la toile, finit notre art sur terre.

- Et, de là, il va se perdre dans les cieux, dit Poussin. ${ }^{29}$

Le tableau de Frenhofer marquait peut être une fin de l'art, mais annonçait aussi un recommencement.

Q uand Borduas parlait semblablement de la « bourgade plastique » comme une « place fortifiée mais trop facile d'évitement »,30 il ne fau-

28. L. Hilberseimer et H. DeARstyne, K. Malevich. The non-objective world, Chicago, P. Theobald, 1959, traduction anglaise d'un essai de $M$ alevitch paru d'abord en allemand sous le titre $D$ ie gegenstandslose Welt, , M unich, A. Langen (Bauhausbücher $n^{0} 11$ ), 1927. M a traduction.

29. H. de BALZAC, op. cit., p. 67-8.

30. «Refus global», dans André-G. BourasSA, Serge Fisette et Gilles LAPoINTE, Paul-É mile Borduas Écrits I, , M ontréal, Les Presses de I'Université de M ontréal, 1987, p. 341. 
drait pas y voir une volonté de revenir au discours et de porter sur le terrain de l'art des préoccupations politiques. Certes, il y a des artistes pour qui l'art est une drogue, comme disait Degas, et qui sont dépourvus de toute conscience sociale. Borduas souhaitait au contraire que les artistes s'engagent, prennent position sur les grands problèmes del'heure, mais il ne demandait pas pour autant qu'ils adhèrent au réalisme social des peintres marxistes. À vrai dire, il avait en horreur toute « peinture de propagande » et parlait volontiers de l'échec de l'art mexicain, de l'art russe et de l'art américain contemporains. ${ }^{31} \mathrm{~A}$ u moment où il exprimait ces jugements, il ne connaissait pas encore l'expressionnisme abstrait américain, mais pensait à Thomas $\mathrm{H}$ art Benton, Ben Shahn et consorts. Pour Borduas, la propagande est bien le pire des discours et la faire taire, un impératif catégorique!

Toutes ces prises de position ont cependant un revers. Le silence des oeuvres se paie de la solitude des artistes, qui ne se réclament plus d'une communauté particulière, mais cherchent à rejoindre l'universel. Certains d'entre eux ne s'y sont résolus qu'avec peine. A insi, Van Gogh rêvait d'établir un «A telier du Sud » à Arles et il comptait sur la présence de $G$ auguin pour donner du prestige à son projet. Sur son invitation pressante, G auguin était venu vivre dans la maison jaune de Vincent à Arles en octobre 1888. O n sait que l'expérience finira mal, Gauguin fuyant A rles persuadé que Vincent était devenu fou et qu'il menaçait de letuer. L'A telier du Sud restera à l'état de projet, une sorte de commune d'artistes avant la lettre.

II existe en Israël, près de $\mathrm{H}$ aïfa, un véritable village d'artiste, Ein Hod, fondé par M arcel Janco (1895-1984), qui participa au mouvement dadaïste à ses débuts. Certes, le projet s'est beaucoup commercialisé avec le temps, surtout à la suite de l'établissement d'une manufacture de tapisseries qui exécutent les cartons de Janco. II n'en reste pas moins qu'Ein $\mathrm{H}$ od fut une tentative de constituer, sur le modèle des kibbutzim, une véritable communauté d'artistes.

31. Voir « Lettre de Borduas à J osephine $\mathrm{H}$ ambleton, de la fin de décembre 1946 » dans André-G. BoURASSA et Gilles LAPOINTE, Paul-É mile Borduas Écrits II, tome 1 : 1923-1953, M ontréal, Les Presses de I'Université de M ontréal, 1993, p. 188-192: "Je considère toutes ces oeuvres comme futiles, illusoires ou grossières. Et les prétentions de ces fabricants, indéfendables ». 
Chez les surréal istes aussi, on trouve un besoin de communauté, voire même la nécessité de définir un « mythe nouveau » pour notre temps. C'est à l'occasion de la $\mathrm{VI}^{\mathrm{e}}$ Exposition internationale du surréalisme, organisée par André Breton et $M$ arcel Duchamp avec le concours de l'architecte Frédéric K iesler à la galerie $M$ aeght à Paris, du 7 juillet au 20 ou 30 septembre 1947, que cette volonté fut le plus clairement exprimée. Breton avait souhaité en effet que cette exposition marquât «par rapport aux précédentes manifestations d'ensemble du surréalisme [...] un certain dépassement » et que ce dépassement se fasse « dans la direction d'un mythe nouveau ». Concrètement, l'exposition avait été conçue sous le mode d'une traversée de plusieurs salles comportant ce que nous appellerions aujourd'hui des « installations ». Le spectateur pénétrait d'abord dans la Salle des Superstitions, dessinée par Kiesler et occupée par les œuvres de $M$ arcel Duchamp (qui y avait son Rayon vert ), $M$ ax Ernst, $\mathrm{M}$ atta, $\mathrm{M}$ iro, Tanguy et les sculpteurs $\mathrm{D}$ avid $\mathrm{H}$ are et $\mathrm{M}$ aria $M$ artins. On passait ensuite dans la « Salle de pluieet de dédale », conçue par $M$ arcel Duchamp, où levisiteur devait se faufiler entre des rideaux de pluies multicolores, sans déranger des joueurs de billard installés au milieu de la salle, pour atteindre enfin les « autels » dressés en autant d'alvéoles octogonales - une douzaineen tout - et consacrés à des divinités aussi peu orthodoxes que le Tigre mondain, ${ }^{32}$ la Chevelure de Falmer , I'H éloderme, J eanne Sabrenas, le Soigneur de Gravité ( partie restée secrète du Grand Verre de Duchamp), etc...Breton avait consacré son « autel » à Léonie Aubois d'A sby, « l'une des plus mystérieuses passantes qui traversent les Illuminations ${ }^{33}$ de Rimbaud. Certes, ce programme n'était pas sans faire problèmeà certains esprits. Breton pouvait craindreque le parcours initiatique imposé aux spectateurs, leculteà rendre à ces divinités nouveau genre, l'idée même de leur dresser des « autels » ne pouvaient pas ne pas évoquer pour eux jene sais quel relent dereligion sinon de bondieuserie. II tentait de prévenir l'objection en proclamant haut et net que « les surréalistes [...] n'ont cessé de se réclamer de la libre pensée intégrale ${ }^{34}$ || ne fallait donc pas entendre cette idée d'un

32. Allusion au conte de ean FERRY, L eTigre mondain, célébré par Breton. On sait que J ules Ferry était le scénariste du célèbre film, Q uai des orfèvres. 33. André BRETON, «Flagrant délit », dans La Clé des champs, Paris, Les Éditions du Sagittaire, 1953, p. 140.

34. André BRETON, "Comète surréaliste», dans La Clé des champs, p. 103. C'est Breton qui souligne. 
« mythe nouveau » comme un simple retour au sentiment religieux d'un autre âge. Dans l'idée d'un «mythe nouveau », l'accent portait sur « nouveau ». N 'empêche que Breton était tenté d'emprunter aux structures archaïques quelques secrets de leur efficacité, leur pouvoir de communion n'étant pas leur moindre attrait.

Claude $G$ auvreau de son côté empruntera à Pierre $M$ abille l'idée d' « égrégore » pour désigner le groupe automatiste québécois, voulant signifier par là que le groupe constituait une unité qui transcendait les individus le composant. On peut donc y voir aussi une nostal gie de vie communautaire mise en péril par l'individualisme de la pure recherche formelle, propre à la peinture moderne.

II y a une autre raison pour laquelle le silence n'a pas de prix en art moderne. II a été la clé qui a ouvert à nos contemporains la porte aux arts dits primitifs, archaïques ou étrangers, parce que leur contenu plus ou moins obscur à nos cultures ne posait plus d'obstacle à notre appréciation de leur forme. Des échanges stylistiques étaient possibles, d'ailleurs dans les deux sens, non seulement de l'O ccident vers les cultures étrangères, mais des cultures étrangères vers I' 0 ccident. L'importance donnée à la forme en art moderne rendait possible une extraordinaire fraternité de toutes les cultures sur le plan esthétique. Le dévot $C$ hrist de Perpignan ( $X$ Ve $\mathrm{s}$.) se révèlait une parenté troublante avec tel masque sénoufo de la Côte d'ivoire. Ces comparaisons, qui auraient été impossibles à faire même au temps de Gauguin, sont devenues monnaie courante depuis que $M$ alraux les a mises à la mode dans ses « musées imaginaires ». Elles ont été magistralement explorées récemment dans une grande exposition du $M$ usée d'art moderne à $\mathrm{N}$ ew York, Primitivism in $20^{\text {th }}$ Century Art. Affinity of the Tribal and the Modern, 1984.

N ous évoquions au début de cet article les premiers missionnaires jésuites de la $\mathrm{N}$ ouvelle-France. O n pourra mesurer la distance parcourue en prenant connaissance de la manière dont ils parlaient de l'art amérindien. Bien souvent ils en niaient purement et simplement l'existence. Le père Pierre Biard croyait avoir trouvé dans leurs conditions de vie, qu'il jugeait misérables, la raison de cette supposée absence.

II est asseuré, écrivait-il, que les disettes grandes suffoquent l'esprit (...) $\mathrm{N}$ ous voyons cecy en nos pauvres sauvages, lesquels, pour ne vivre qu'au jour à la journée et par conséquent tousjours asservis à la crainte de la faim, première et plus forte nécessité de toutes, n'ont moyen de cul- 
tiver leur esprit en la recherche des sciences, non pas mesme de se pourvoir des arts et industries pour l'aisance et l'amélioration de la vie, ny pour fournir aux autres défauts, quoy que bien pressants. A ceste cause donc, ils manquent [...] de toutes lettres et beaux artifices. ${ }^{35}$

Q uand il leur arrivait malgré tout de rencontrer quelques exemples authentiques d'art amérindien, ils n'y voyaient que des « grotesques » ou des « marmousets » comme par exemple, le père Lemercier parlant d'une pratique des Algonquiens de l'O uest.

Ils ont un marmouset de bronse noir, pris sur le païs, qui a un pied de haut ; auquel il donne de la barbe, comme à un European ; quoy que les Sauvages n'en ayent point. II y a certains jours destinez pour honorer cette statuë, par des festins, par des jeux, par des danses, \& mesme par des prieres qu'ils luy adressent, avec diverses ceremonies. II y en une entre-autres, qui est de soy ridicule ; mais qui est remarquable, en ce qu'elle contient une espece de sacrifice. Tous les hommes, les uns après les autres, s'approchent de la statuë, \& pour luy rendre hommage de leur tabac, ils luy presentent la pipe en main, pour petuner : mais comme l'idole ne peut s'en servir ; ils petunent en sa place, luy rejetant au visage la fumée du tabac qu'ils ont dans la bouche; ce qui peut passer pour une espece d'encensement, $\&$ de sacrifice.

O n sait que le mot marmouset était employé en France à l'époque pour désigner de petites figures sculptées, accroupies ou couchées, formant la décoration des abouts de poutres ou remplissant les espaces vides des caissons au plafond des églises. Le mot lui-même dérive de « marmonner», onomatopée pour « bredouiller», dire des choses incompréhensibles. Le moins que l'on puisse dire est que désigner une oeuvre d'art du nom de marmouset n'était pas très élogieux. Surtout c'était faire preuve d'une grande fermeture à l'art des autres cultures, à tout ce qui ne relèvait pas de la sienne. Le jugement porté est d'autant plus paradoxal qu'il s'étend même aux comportements de ces Algonquiens à l'égard de leur image, comportements qui, pourtant, n'étaient pas si différents de ceux qui avaient cours en chrétienté, puisqu'on pouvait y voir « une espece d'encensement ». Ces comportements avaient le tort d'être ceux des autres. Ils ne méritaient pas plus d'égard que ceux que la Bible attribuait aux « nations » ayant le culte des idoles, tel qu'on peut le lire dans Jérémie.

35. «Relation de 1616 » dans Lucien CAMPEAU, Monumenta Novae Franciae, Q uébec, Presses de l'Université Laval, 1967, p. 499. 
O ui, la Terreur des peuples est un pur néant ;

ce n'est que du bois coupé dans une forêt,

travaillé par le sculpteur, ciseau à la main, puis enjolivé d'argent et d'or

[...] tous ne sont qu'oeuvres d'artistes.

On les habille de pourpre violette, on les fixe

pour qu'ils ne bougent pas.

Tels un épouvantail dans une melonnière, ils ne parlent pas ;

il faut les porter, car ils ne marchent pas!

$\mathrm{N}$ 'en ayez pas peur : ils ne peuvent faire du mal,

et du bien pas davantage ${ }^{36}$.

Il est ironique dans la circonstance que les rituels amérindiens comportaient de la fumée ( faisant donc penser à l'encens), mais investissaient la zone orale d'une manière si nouvelle par rapport aux façons perçues comme «normales » (parole et nourriture) en 0 ccident que leur affinité avec les images parlantes ne furent même pas soupçonnée. Lévi-Strauss explique que pour les A mérindiens, I'usage du tabac est ultra-culinaire, en ce sens que c'est une nourriture qui doit être brûlée pour être consommée. $M$ ais il y a plus. II leur arrivait comme chez les Bororo de distinguer entre deux techniques de la consommation du tabac. Dans un cas, on exhale la fumée, - c'est bien ce qui se passait dans notre exemple algonquien - , et de cette manière, on établit une communication bénéfique avec les Esprits. Dans l'autre cas, on ingère la fumée, ce qui entraîne la transformation des humains en animaux. ${ }^{37}$ Le tabac qu'on exhale sert à une sorte de communication et d'échange avec les Esprits. II se rapproche donc de la parole, alors que celui qu'on ingère se comporte comme un aliment, voire comme une drogue ou un poison. Dans le mythe analysé par Lévi-Strauss, l'ennemi auquel le héros donne le « mauvais tabac » est transformé en tamanoir, cet animal «bouché » par les deux bouts, sans bouche et sans anus. Par contre, l'idole dans le visage de laquelle on exhale de la fumée est à la fois un dieu à qui l'on parle et qui répond à son tour. Serions-nous si loin des images parlantes?

36. Jr 10, 3-4.

37. Claude LÉVI-Strauss, D u miel aux cendres, Paris, Plon, 1966, p. 52-53. 
Les missionnaires auraient refusé de nous suivre jusque là. Si les idoles sont des images parlantes, leurs paroles ne peuvent être qu'illusoires et ceux qui l'entendent sont certainement trompés. Confrontés chez les Indiens à des manifestations qu'ils jugeaient surnaturelles, les missionnaires avaient tôt fait d'évoquer le père du mensonge, le diable. Le même père Biard que nous citions plus haut n'avait aucun doute à ce sujet.

...touteleur religion, pour le dire en un mot, n'est autre qu'ès sorcelleries et charmes des autmoins [ = sorciers ...]. Ils ont beaucoup d'autres semblables sacrifices faits au diable, pour avoir bonne fortune à la chasse, pour la victoire, pour le bon vent, etc. Ils croient aussi aux songes, afin que nulle sorte de folie ne leur manque. ${ }^{38}$

C'est d'ailleurs cette conviction qui justifie - du moins à leurs yeux - les comportements des missionnaires à l'égard des « idoles » amérindiennes. Ainsi quand les pères $A$ llouez et $D$ ablon découvrirent « comme une idole que les Sauvages honorent » près des rapides de la rivière aux R enards, ils n'eurent de cesse que de la détruire. "Pour oster cette occasion d'idolatrie, nous l'avons fait enlever, à force de bras, \& l'avons jetté au fond de la rivière, pour ne paroistre plus jamais ». ${ }^{39} \hat{A}$ une autre occasion, à propos d' « une grande roche taillée grossièrement en statue d'homme », Allouez se contente de noter laconiquement : " $\mathrm{N}$ ous la roulâmes dans l'eau ».40

N ous voilà donc au coeur du problème. Le pari formel de l'art moderne, ou si l'on veut son silence, a été la condition de son ouverture aux formes d'art autres. Cette ouverture vaut cent fois mieux que les anciennes attitudes d'intolérance, mais est-elle sans problèmes? On ne peut s'empêcher de penser que cette appréciation purement esthétique des formes d'art exotiques ne constitue qu'une toute première approche de leur signification et bien souvent elle ne permet pas d'aller plus loin que l'affirmation d'un certain spiritualisme perçu (projeté?) à travers ses formes. Dieu sait, par exemple, comment la peintre canadienne Emily Carr était sensible à l'art des Indiens de la Côte $O$ uest. Voulant dire ce qu'elle avait perçu dans leur art et tenté d'exprimer dans ses propres peintures, elle s'en tenait à des formules

38. L. Cam Peau, op. cit., p. 506.

39. Relation de 1670 ; J es Rel., vol. 55, p. 192.

40. Relation de 1672-1673; J Jes. Rel., vol. 58, p. 42. 
qu'on aurait pu trouver dans Edmund Burke, auteur d'une Philosophical Enquiry into the $O$ rigins of $O$ ur I deas of the Sublime and the Beautiful, 1756 ou dans Goethe.

N ext timel paint Indians I'm going off on a tangent tear. There is something bigger than fact : the underlying spirit, all its stands for, the mood, the vastness, the wildness, the Western breath of go-to-the devil-if-you-don't-likeit, the eternal big spaceness of it. $\mathrm{O} h$ the West! I' $m$ of it and I love it. ${ }^{41}$

Ce qui n'est pas perçu dans ce genre de caractérisations, c'est la relation de ces autres cultures à l'Univers et la fonction que jouaient les objets que nous percevons comme des oeuvres d'art dans cette relation. L'appréciation purement esthétique que nous en faisons permet leur déplacement hors de leurs milieux d'origine. $\mathrm{N}$ ous les retrouvons dans nos musées et dans nos collections privées. Ils se frayent un chemin jusque dans les oeuvres de nos artistes, comme la trace de ces yeux en cylindres d'un masque Grebo de la Côte d'ivoire dans une Guitare, 1912 de Picasso. Le langage universel des formes s'accompagne d'un déracinement des oeuvres, de leur mise hors contexte.

Q u'on explore le silence de la peinture moderne comme une réclamation des droits de l'individu contre toutes les idéologies totalitaires, ou comme une ouverture sur des mondes autres, on trouve aussi son besoin d'enracinement, de communauté frustré, ou sa compréhension del'autre limitée. Cequ'on gagne par un bout, on semble le perdre par l'autre. La liberté et l'ouverture restent toutefois des biens inaliénables. M ême si une nouvelle synthèse paraît parfois souhaitable, elle ne pourra se faire sans eux. Ils auront toujours la première place. II ne saurait être question de revenir en arrière.

\footnotetext{
RÉSUMÉ

Rien n'est plus silencieux qu'un tableau. Une longue évolution a conduit à ces silences volontaires. On trace ici ce parcours, depuis l'iconographie religieuse « parlante » en passant par le travail des surréalistes, l'art abstrait et la peinture contemporaine. De la fenêtre sur le monde au tableau-mur les artistes ont dû lutter pour que leur art ne serve plus les intérêts des puissants, mais qu'il conduise au silence et au questionnement.
}

41. Emily CARR, notation du 14 novembre 1927 dans $H$ undreds and Thousands. The J ournal of Emily Carr, Toronto/Vancouver, Clarke, Irwin \& Company Limited, 1966, p. 5. 


\section{ABSTRACT}

There is nothing more silent than a painting. A long evolution has lead to these voluntary silences. We intend here to trace back this movement, from the "talkative" religious iconography, to the work of the surrealists, to abstract art up to contemporary painting. From the window on the world to the painting-wall, artists had to fight hard so that their art would not serve the interests of the powerful, but would lead to silence and a requestioning. 